\title{
3D non-affine finite strains measured in isolated bovine annulus fibrosus tissue samples
}

\author{
J. M. Huyghe • C. J. M. Jongeneelen
}

Received: 22 August 2010 / Accepted: 4 March 2011 / Published online: 31 March 2011

(C) The Author(s) 2011. This article is published with open access at Springerlink.com

\begin{abstract}
Understanding of the mechanics of disc tissue calls for measurement of strains in physiological conditions. Because the intervertebral disc is gripped between two vertebrae, the swelling is constrained in vivo, resulting in a intradiscal pressure of $0.1-0.2 \mathrm{MPa}$ in supine position. The excision of isolated disc tissue samples results often in nonphysiological swelling. The purpose of the present study is to measure 3D finite strains in isolated bovine disc tissue specimens under physiological osmolarity and pressure, particularly around discontinuities of the collagen network. The collagen is stained by means of CNA35 probe, and the (dead) cells are stained by means of propidium iodide. The tissue is observed under confocal microscopy, under an externally applied pressure generated by a PEG solution. The 3D finite strains are obtained through correlation of the texture of the 3D images. The correlation technique yields principal strains in all areas except within collagen-free areas. The deformation is strongly non-affine. Especially around discontinuities, the strain field is non-homogeneous. Macroscopic strains as computed from finite element analysis of whole discs are insufficient to predict microstrains around clefts or cells. Because of the small number of specimens, the present results should be considered preliminary.
\end{abstract}

Keywords Intervertebral disc - Osmolarity · Confocal microscopy $\cdot$ Correlation

\section{J. M. Huyghe $(\varangle)$}

Department of Biomedical Engineering,

Eindhoven University of Technology, P.O. Box 513,

5600 MB Eindhoven, The Netherlands

e-mail: j.m.r.huyghe@tue.nl

C. J. M. Jongeneelen

Department of Mechanical Engineering,

Eindhoven University of Technology, P.O. Box 513,

5600 MB Eindhoven, The Netherlands

\author{
List of symbols \\ A Second order tensor \\ a Vector \\ $\mathbf{a} \cdot \mathbf{b} \quad$ Inner product of vector $\mathbf{a}$ and $\mathbf{b}$ \\ $\mathbf{A}^{C} \quad$ Conjugate of second order tensor $\mathbf{A}$ \\ A : B Double dot product $(=\operatorname{tr}(\mathbf{A} \cdot \mathbf{B}))$ \\ E Strain tensor of the solid \\ I Unity tensor \\ $c^{s} \quad$ External salt concentration \\ $p \quad$ Pressure \\ F Deformation gradient tensor \\ $R \quad$ Universal gas constant \\ $T$ Temperature \\ PG Proteoglycans \\ PEG Polyethylene glycol \\ PI Propidium iodide
}

\section{Introduction}

Intervertebral disc tissue consists of a fluid-filled extracellular matrix, in which living cells are sparsely dispersed. The mechanical function is highly dependent on the composition of the extra-cellular matrix, which primary consists of collagen fibrils and negatively charged proteoglycans. Due to the fixed charges of the proteoglycans (PGs), the cation concentration inside the tissue is higher than physiological. This excess of ion particles leads to an osmotic pressure difference, which causes swelling of the tissue (Urban and Maroudas 1979). Because the intervertebral disc is gripped between two vertebrae, the swelling is constrained in vivo, resulting in a intradiscal pressure of 0.1-0.2 MPa in supine position (Wilke et al. 2001). It has been shown that the osmotic pressure inside cartilaginous tissues is much higher than would be expected based on its FCD 
(Urban and McMullin 1988). This is because part of the water in the tissue is absorbed by the collagen fibres. The proteoglycan molecules, because of their large size, are excluded from this intrafibrillar space. This means that their effective concentrations are much higher in the extra-fibrillar space than if they were distributed uniformly throughout the entire matrix. Hence, the effective fixed charge density is higher than if computed from total tissue water content. A recent study demonstrates that intrafibrillar water increases osmolarity within the annulus fibrosus substantially (Schroeder et al. 2007). On the other hand, Wognum et al. (2006) show by means of two different models of the disc that high osmolarity within the disc may have a protective effect against crack propagation within the disc. Hence, the decrease in osmolarity, associated with degeneration, may be an explanation of (1) the growing number of cracks observed in the degenerating disc as well as (2) the poor correlation between external loading and crack propagation (Videman and Nurmunen 2004). The sensitivity of disc cells (Wuertz et al. 2007), cracks (Wognum et al. 2006) and intrafibrillar water (Sivan et al. 2006) to changes in osmolarity makes physiological osmolarity conditions a must if an in vitro isolated disc tissue experiment is to represent the in vivo conditions. In order to get insight into the internal mechanics of the disc, a combination of finite element analysis (Shirazi-Adl et al. 1984; Iatridis et al. 2003; Schroeder et al. 2007) and experimental measurement is the preferred approach. Experimental measurement of vertebral displacements in motion segments (Heuer et al. 2007), of stress-strain relationships of isolated tissue experiments (Best et al. 1994; Houben et al. 1997; Huyghe et al. 2003; Bass et al. 2004; Perie et al. 2004), of outer annulus strain (Bruelmann et al. 2004a,b) and of intradiscal pressure (Wilke et al. 2001) are within reach. Finite element models, fitted to a combination of different experimental data, yield quantitative data of those variables that are more difficult to measure, such as 3D stress components. The use of isolated tissue specimens, however, presents the danger of testing the tissue in unphysiological conditions. Particularly, the osmotic prestress is a critical issue. Submersion of the tissue samples in physiological salt solution typically results in unphysiological swelling. The unphysiological water inhibition may in turn lead to unphysiological intrafibrillar water content or unphysiological cellular responses. In situ measurements are a way to circumvent this problem (Bruelmann et al. 2004a,b; Heuer et al. 2007). However, to the best of our knowledge, present day in situ methods are restricted to $2 \mathrm{D}$ finite strain measurements. The purpose of the present study is to measure $3 \mathrm{D}$ finite strains in isolated bovine disc tissue specimens under physiological osmolarity and pressure, particularly around discontinuities of the collagen network. Because of the small number of specimens involved, this study should be seen as a preliminary report of a technique, not a full study.

\section{Materials and methods}

\subsection{Specimen preparation}

A bovine tail from a 20-24 months old cow was obtained from a local butcher. The four upper coccygeal intervertebral discs (CC1-CC2, CC2-CC3, CC3-CC4, CC4-CC5) were dissected from the adjacent vertebral bodies by cutting as close as possible to the end-plates. They were snap frozen and stored in $-80^{\circ} \mathrm{C}$ until used. Because of the exploratory nature of the present study, we do not keep the cells alive in the tissue samples. From the frozen disc, the nucleus was removed and the annulus was sliced in about $1 \mathrm{~mm}$ thick samples perpendicular to axial direction by hand with a scalpel. The resulting 4 tissue samples, about $4 \mathrm{~mm}$ by $7 \mathrm{~mm}$ in size, were taken from the posterior annulus fibrosus and were placed in phosphate-buffered saline (PBS) for $1 \mathrm{~h}$ to rehydrate. Subsequently, these four annulus samples were incubated at $37^{\circ} \mathrm{C}$ overnight in PBS containing a final concentration of $8 \mu \mathrm{M}$ CNA35OG488. The recently developed CNA35-OG488 probe binds to a wide range of collagen (type I to VI) with different fluorescent intensities and has little cross-reactivity with non-collagenous extra-cellular matrix proteins (Krahn et al. 2006). The CNA35 probe is Oregon Green-labelled and is used to visualize the extra-cellular matrix. For the calculations of the strains, the fibre displacements were measured. Propidium iodide was used to visualize (dead) cells in the tissue to locate the cells.

\subsection{The osmotic loading chamber}

The tissue was placed on top of the objective glass in $0.15 \mathrm{M}$ $\mathrm{NaCl}$ (Fig. 1). A membrane with a diameter of $30 \mathrm{~mm}$ was cut out of a dialysis tubing (MW cutoff 8,000, Spectrapor, Los Angeles, USA) and placed over the tissue (Fig. 1). The membrane was tightened between two rings to prevent the chamber from leaking. A Zeiss LSM 510 confocal laser scanning microscope (CLSM) with a $40 \times$ LD ACHROPLAN/0.6 corr lens was used to visualize the tissue deformation. Thirty-three 3D images were made. Each image was typically $230.3 \times 230.3 \times 29.1 \mu \mathrm{m}$ with a resolution of $512 \times 512 \times 21$ pixels. To improve the resolution, the images were made by averaging the scanning 4 times. One three-dimensional image took $15 \mathrm{~min}$ to scan. Every $20 \mathrm{~min}$, one image was harvested. Polyethylene glycol (PEG) 20,000 (Fluka BioChemika Ultra, St. Gallen, Switzerland) diluted in $0.15 \mathrm{M} \mathrm{NaCl}$ was placed above the membrane (Basser et al. 1998; Ishihara et al. 1996). Different PEG concentrations were used. The PEG calibration curve of Maroudas et al. (1991) translated the PEG concentration into an osmotic pressure. As suggested by Maroudas et al. (1991), the temperature was kept constant at $25^{\circ} \mathrm{C}$, using a temperature control device. 


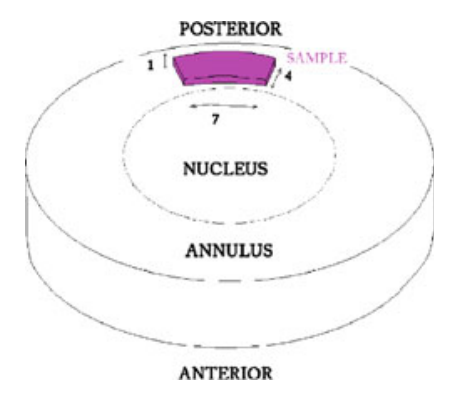

(a)
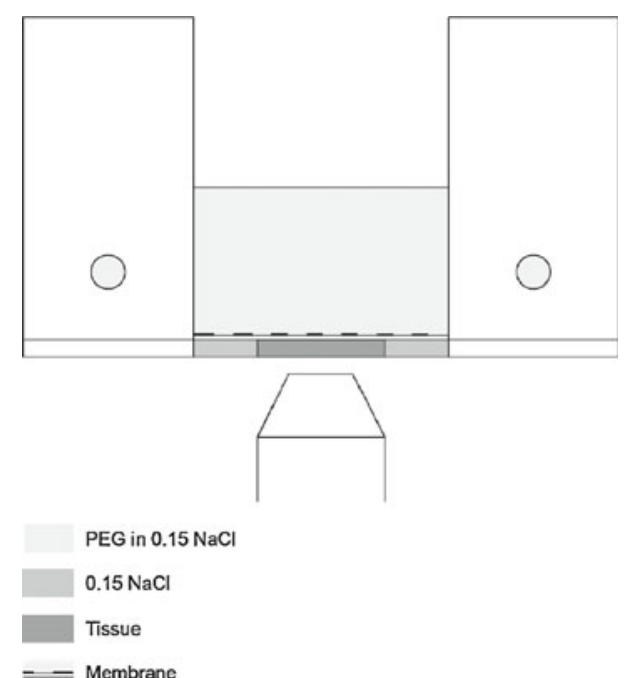

(b)

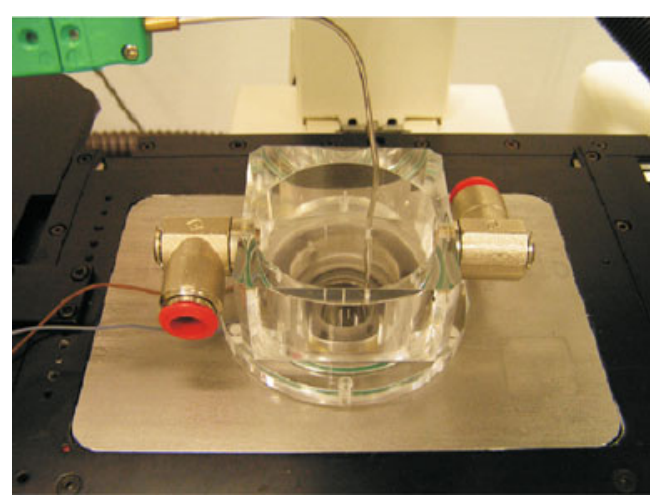

(c)

Fig. 1 The osmotic loading chamber. The tissue is in the chamber under the membrane. The PEG is on top of the membrane. The membrane is permeable to water and impermeable to PEG and PG. a Samples are extracted from the posterior annulus fibrosus of the coccygeal discs of an adult cow. $\mathbf{b}$ Schematic representation of the tissue chamber. The circles on the side are led lights to regulate the fluid temperature. c A picture of the set-up. A thermo-couple measures the temperature which serves as a feedback to the led heating

\subsection{Loading by means of a PEG solution}

The equilibrium of the tissue with a external solution requires:
$p-\pi_{\mathrm{PG}}=-\pi_{\mathrm{PEG}}-2 R T c^{s}$

in which $R$ represents the universal gas constant $(8.314$ $\mathrm{J} \mathrm{K}^{-1} \mathrm{~mol}^{-1}$ ), T the absolute temperature $(\mathrm{K}), c^{s}$ is the saline concentration $(0.15 \mathrm{M})$ and $\pi_{\mathrm{PEG}}$ is the osmotic pressure associated with the PEG. For the displacement experiment, a PEG concentration of $10 \mathrm{~g} / 100 \mathrm{~g}$ saline was used, which creates a $\pi_{\mathrm{PEG}}$ of approximately $0.1 \mathrm{MPa}$ (Maroudas et al. 1991).

\subsection{Three-dimensional digital image correlation technique}

A three-dimensional digital image correlation (DIC) was used to calculate the displacements (Verhulp et al. 2004). The method used is based on the two-dimensional technique developed by Sutton et al. (1986). Verhulp et al. (2004), Hendriks (2005) have extended this two-dimensional technique to three dimensions for strain measurements. The detailed description of the method is found elsewhere (Verhulp et al. 2004). Briefly, when an image is digitized, the intensity pattern of the reflected light is stored as grey values. Each small subset of grey values in the reference situation is related to a small subset in the deformed situation. Linear transformation of the reference subset to the deformed subset is assumed:

$$
\begin{gathered}
x^{\prime}-x=u+\frac{\partial u}{\partial x} \mathrm{~d} x+\frac{\partial u}{\partial y} \mathrm{~d} y+\frac{\partial u}{\partial z} \mathrm{~d} z \\
y^{\prime}-y=v+\frac{\partial v}{\partial x} \mathrm{~d} x+\frac{\partial v}{\partial y} \mathrm{~d} y+\frac{\partial v}{\partial z} \mathrm{~d} z \\
z^{\prime}-z=u+\frac{\partial w}{\partial x} \mathrm{~d} x+\frac{\partial w}{\partial y} \mathrm{~d} y+\frac{\partial w}{\partial z} \mathrm{~d} z
\end{gathered}
$$

where $(x, y, z)$ is the reference position of a point, $\left(x^{\prime}, y^{\prime}, z^{\prime}\right)$ is the deformed position of that point, $(u, v, w)$ is the displacement of the centre of a subset and $(\mathrm{d} x, \mathrm{~d} y, \mathrm{~d} z)$ represent the distance from the centre of the subset to the point $(x, y, z)$.

When a reference subset $A(x, y, z)$ is compared to a deformed subset $B\left(x^{\prime}, y^{\prime}, z^{\prime}\right)$, the optimized image correlation technique maximizes the correlation coefficient $R$ by minimizing the function:

$$
1-\frac{\sum_{i, j, k=1}^{N} A\left(x_{i}, y_{j}, z_{k}\right) \sum_{i, j, k=1}^{N} B\left(x_{i}^{\prime}, y_{j}^{\prime}, z_{k}^{\prime}\right)}{\left(\sum_{i, j, k=1}^{N} A\left(x_{i}, y_{j}, z_{k}\right)^{2} \sum_{i, j, k=1}^{N} B\left(x_{i}^{\prime}, y_{j}^{\prime}, z_{k}^{\prime}\right)^{2}\right)^{\frac{1}{2}}}
$$

which corresponds to a search for the best position and deformation of the subset $A(x, y, z)$ in subset $B\left(x^{\prime}, y^{\prime}, z^{\prime}\right)$. The function $\mathrm{S}$ that must be minimized depends on 12 variables: 
$u, v, w, \frac{\partial u}{\partial x}, \frac{\partial u}{\partial y}, \frac{\partial u}{\partial z}, \frac{\partial v}{\partial x}, \frac{\partial v}{\partial y}, \frac{\partial v}{\partial z}, \frac{\partial w}{\partial x}, \frac{\partial w}{\partial y}, \frac{\partial w}{\partial z}$

To improve the convergence, the correlation takes two steps: (1) The window is assumed to translate without deformation or rotation. The displacement gradients are therefore set to zero, and a Fast Fourier Transformation (FFT) calculation is used to determine the correlation functions for all possible window positions in the search area $(u, v$ and $w$ are determined). (2) An iterative search algorithm is used to determine the location of maximum correlation more accurately. The FFT-calculated displacements $u, v$ and $w$ are used as initial estimates in the Broyden-Fletcher-Goldfarb-Shanno method. The calculation allows the window to deform linearly, and a four-point cubic interpolation is used to evaluate image values between adjacent pixels. This gives the calculations a sub-pixel accuracy. The size of the window and the search area used in the DIC program had to be optimized for each region and time step, but they were all approximately $13 \times 13 \times 13$ and $40 \times 40 \times 40$ pixels for the strain calculations (Fig. 2).

The correlation technique was tested for rigid body displacement, using confocal images obtained from the disc. Image dimensions of 325 by 325 by $30 \mu \mathrm{m}$, corresponding to 512 by 512 by 23 pixels were used in the example shown in Fig. 3. Image correlation yielded a rigid body displacement at $9 \times 4 \times 4=144$ locations in the tissue for 11 pairs of 3D images. Each pair of images represented the image before and after the rigid body displacement. The ratio of standard deviation over average was calculated for each of the 11 measurements.

\subsection{Resolution}

The resolution is meant to express the separate visibility, both laterally and axially, of points during the scanning process. The resolution is based on the Rayleigh criterion. This defines the distance between two sources that can be imaged independently, as the distance at which a maximum of the one Airy pattern (interference pattern) coincides with the first minimum of a second one. The resolution is not the same for all three dimensions. For a numerical aperture $>0.5$, the axial resolution is given by the following equation:

$r_{\mathrm{axial}}=\frac{0.88 \cdot \lambda_{\mathrm{exc}}}{\left(n-\sqrt{n^{2}-\mathrm{NA}^{2}}\right)}$

Where $r_{\text {axial }}$ is the axial resolution, $\lambda_{\text {exc }}$ is the excitation wavelength, $\mathrm{n}$ is the refractive index of the immersion liquid and NA is the numerical aperture (Juskaitis 2006). The lateral resolution is given by

$r_{\text {lateral }}=0.51 \frac{\lambda_{\text {exc }}}{\mathrm{NA}}$.

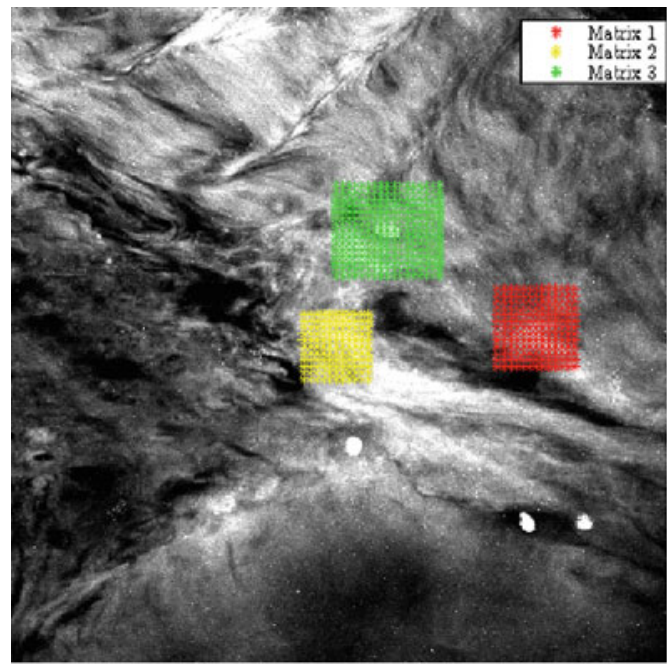

(a)

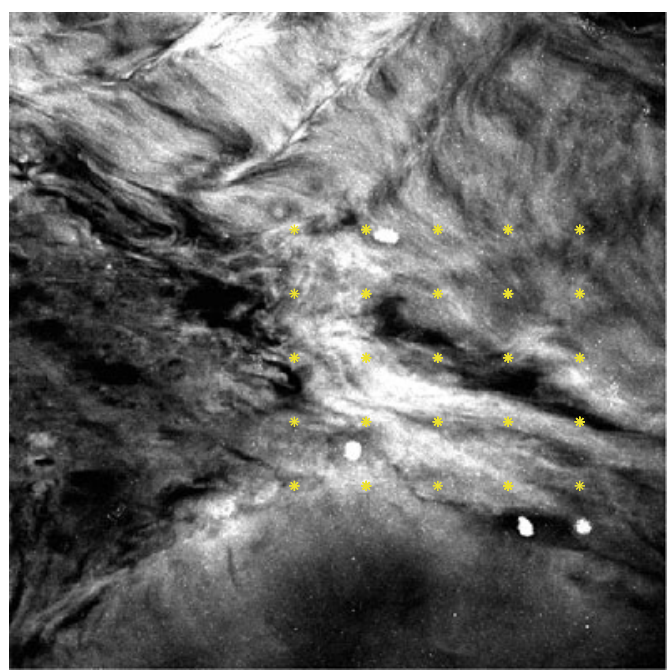

(b)

Fig. 2 A two-dimensional representation of the different regions with marker points used for the image correlations. a Location of the matrix regions used for calculations, Matrix 1 (Red, right square), Matrix 2 (Yellow, lower left square) and Matrix 3 (Green, upper square). b Location of the global grid used for calculations (color figure online)

The lateral resolution has a linear relationship with the numerical aperture (NA) of the lens, whereas the axial resolution varies as the square of the NA. So the axial resolution is in general poorer than the lateral resolution (Table 1).

\subsection{Strain calculations}

The correlation method was used for the strain calculation. A deformation tensor is calculated from the discrete displacement field. A particle $P_{0}$ at time $t=0$ is observed, and the distance to its neighbouring particle $Q_{0}$ is calculated. $P_{0}$ has position vector $\mathbf{x}_{0}$, the position vector of $Q_{0}$ is defined as 


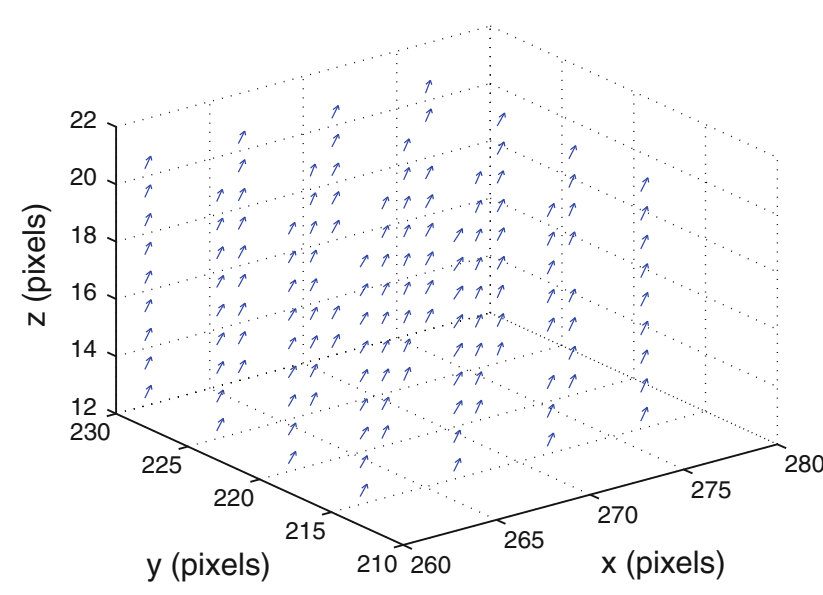

Fig. 3 The displacement field as it was calculated with the image correlation program for a typical experiment

Table 1 Resolution of the images

\begin{tabular}{llll}
\hline Stain & $\lambda_{\text {exc }}(\mathrm{nm})$ & $r_{\text {axial }}(\mu \mathrm{m})$ & $r_{\text {lateral }}(\mu \mathrm{m})$ \\
\hline CNA & 488 & 2.15 & 0.41 \\
\hline
\end{tabular}

$\mathbf{x}_{0}+d \mathbf{x}_{0} . P_{t}$ and $Q_{t}$ are the corresponding particles in the deformed state at time $\mathrm{t}$. The deformation vector in $P_{t}$ at time $\mathrm{t}$ is defined as:

$\mathrm{d} \mathbf{x}_{t}=\mathbf{F} \cdot \mathrm{d} \mathbf{x}_{0}$

resulting in

$\mathbf{F}=\left(\nabla_{0} \mathbf{x}_{t}\right)^{c}=\frac{\partial \mathbf{x}_{t}}{\partial \mathbf{x}_{0}}$

The position of the neighbouring point $\mathbf{x}_{t}+\Delta \mathbf{x}_{t}$ can be written as a Taylor-series expansion:

$\Delta \mathbf{x}_{t}=\frac{\partial \mathbf{x}_{t}}{\partial \mathbf{x}_{0}} \cdot \Delta \mathbf{x}_{0}+\frac{\partial^{2} \mathbf{x}_{t}}{\partial \mathbf{x}_{0}^{2}}: \Delta \mathbf{x}_{0} \Delta \mathbf{x}_{0}+\mathbf{a}$ where $\mathbf{a}$ is the truncation error. A particle $P_{t}$ is surrounded by $k$ neighbouring particles. For each of these neighbouring particles, $\Delta \mathbf{x}_{t}$ is calculated which eventually results in a estimation for the deformation tensor $\mathbf{F}$. The Green-Lagrange strain tensor $\mathbf{E}$ is

$\mathbf{E}=\frac{1}{2}\left(\mathbf{F}^{c} \cdot \mathbf{F}-\mathbf{I}\right)$

where $\mathbf{I}$ is the unit tensor.

\section{Results}

\subsection{Confocal microscopy}

\subsubsection{Rigid body motion}

Rigid body displacements are imposed upon the annulus fibrosus samples. The image correlation is used to calculate the rigid body displacements of eleven different pairs of 3D images of the tissue. The rigid body displacement as calculated by the correlation technique for one of the pairs of images is shown in Fig. 3. The standard deviation of the $\mathrm{x}$-displacement was $0.49 \mu \mathrm{m}$.

\subsubsection{Deformation}

Figure 4 shows the swelling observed with the $40 \times$ objective of the confocal microscope following a reduction in the PEG concentration above the dialysis membrane from 10 to $0 \%$. The collagen fibres were stained with CNA35 probe (green), while the cells were stained with PI (red). Large changes in distance between the cells point at large deformation. The swelling occurs mostly between the lamellae (Fig. 4).
Fig. 4 Images from confocal microscopy. The full Z-stack corresponding to these images are found under Online Resource 1 . a $t=0,10 \%$ PEG in $0.15 \mathrm{M} \mathrm{NaCl}$. b $t=8 \mathrm{~h}, 0 \%$

$0.15 \mathrm{M} \mathrm{NaCl}$

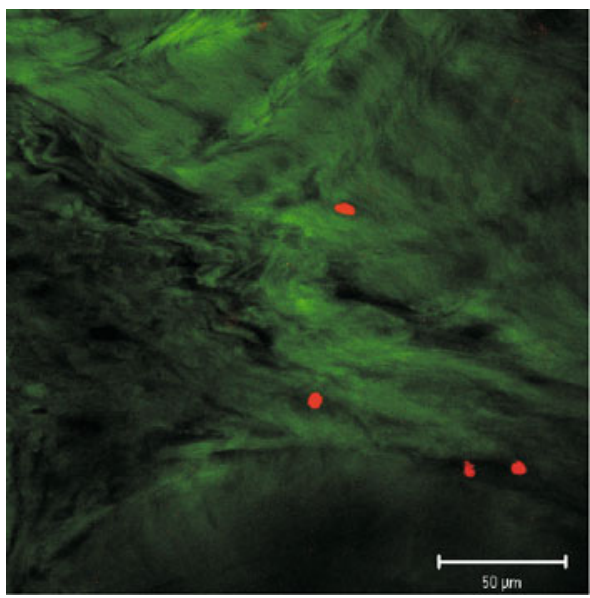

(a)

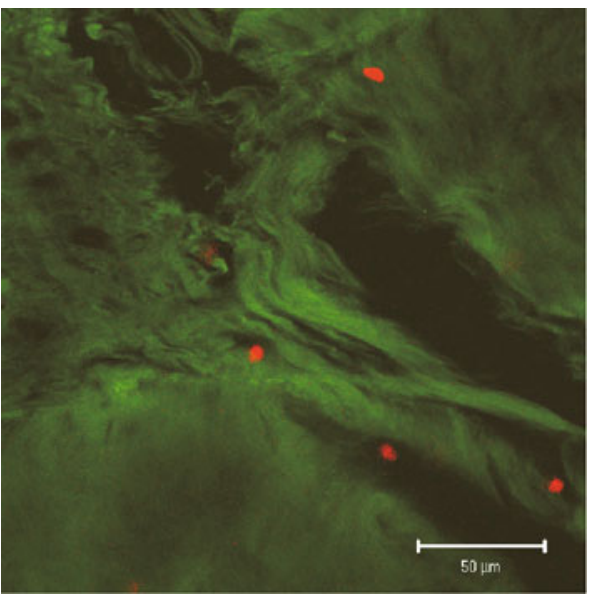

(b) 


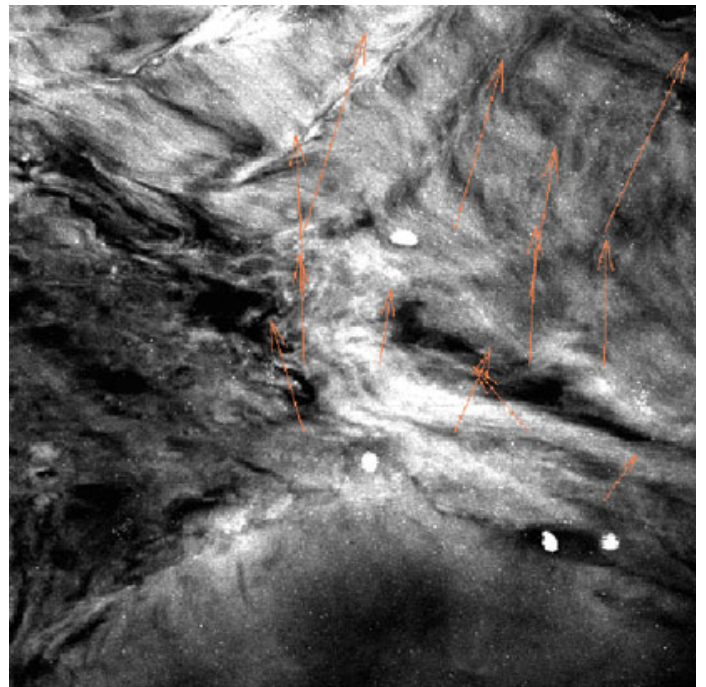

(a)

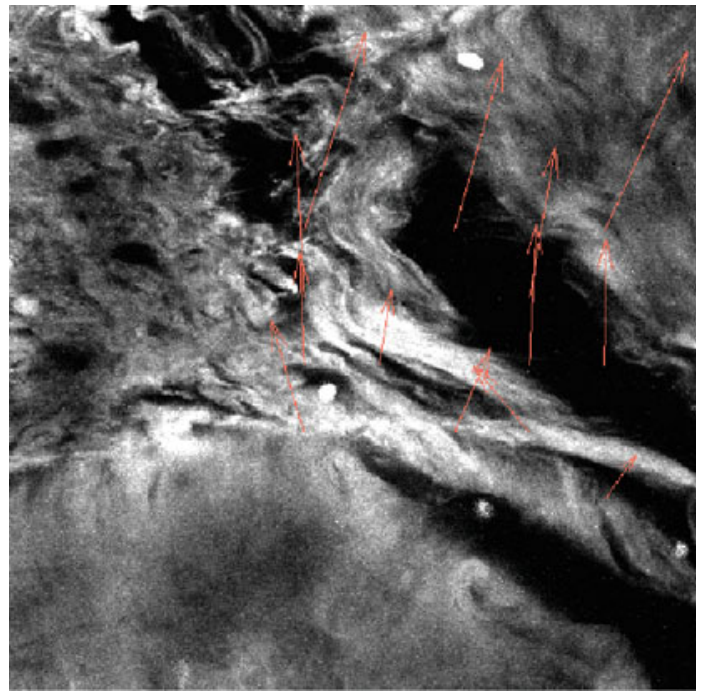

(b)

Fig. 5 2D displacement field of the global grid, calculated by the image correlation. a Displacement vectors starting in the given grid points (Fig. 2b) projected onto the undeformed situation. b Same displacement vectors projected onto the deformed situation

\subsubsection{D Correlation technique}

Figure 2a shows three different extra-cellular matrix regions. Matrix 1 and 3 are in a collagen-rich lamella region. Matrix 3 includes the pericellular environment of cell 1. Matrix 2 is chosen in an interlamellar region. Figure $2 \mathrm{~b}$ shows the global grid. Figure 5 shows the displacement field of the global grid between $t=0$ and $t=8 \mathrm{~h}$. The image correlation technique was not able to find all the points in the deformed state, some points got lost due to the change in contrast in the deformed state. Table 2 shows the percentage of calculated points. All
Table 2 Number of points found with the image correlation

\begin{tabular}{lccc}
\hline Region & $\begin{array}{l}\text { Number of } \\
\text { defined points }\end{array}$ & $\begin{array}{l}\text { Number of } \\
\text { calculated points }\end{array}$ & Percentage \\
\hline Matrix 1 (CNA) & 1,859 & 1,065 & 57 \\
Matrix 2 (CNA) & 1,331 & 494 & 37 \\
Matrix 3 (CNA) & 2,805 & 1,497 & 53 \\
Global & 200 & 49 & 25 \\
\hline
\end{tabular}

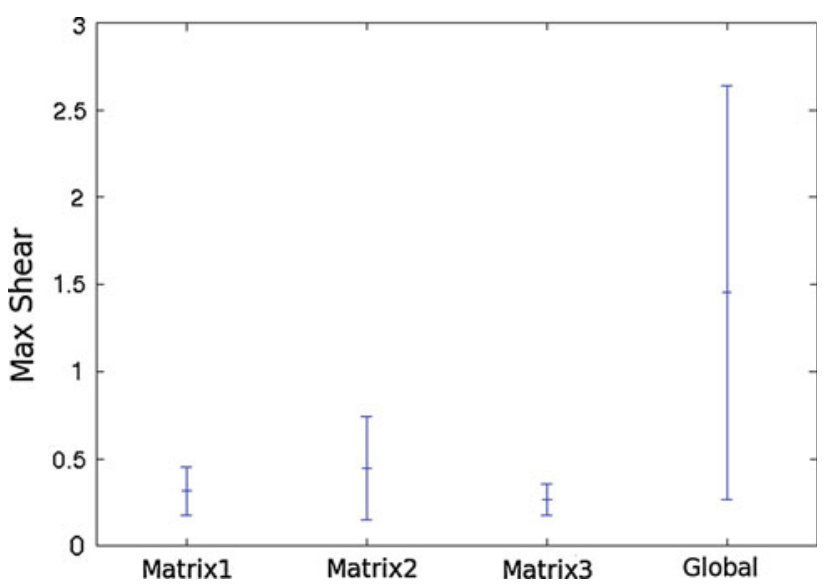

Fig. 6 Average and standard deviation of the maximum shear strain for the separate regions and for the 3D image as a whole

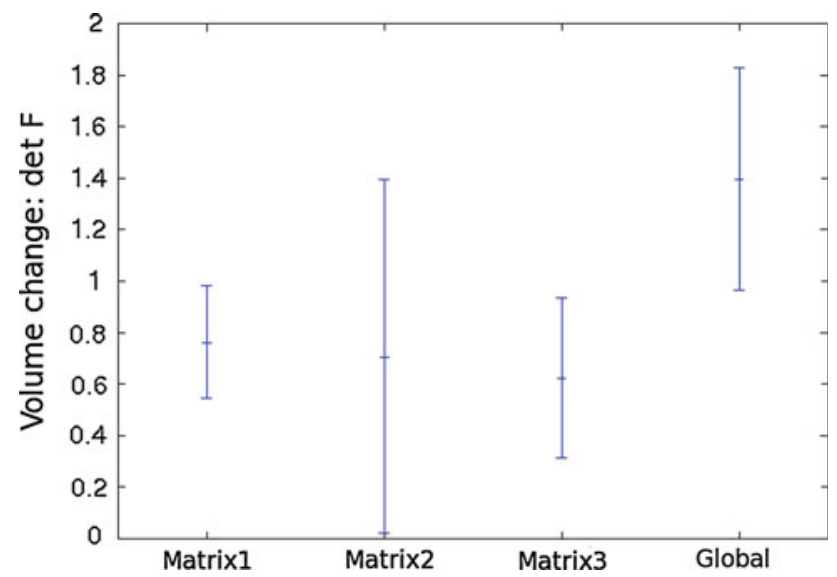

Fig. 7 Average and standard deviation of the determinant of the displacement gradient tensor for the separate regions and for the 3D image as a whole. The determinant represents relative volume change. The local volume of the collagen regions decrease, probably because fluid is exudated from the collagen matrix at the profit of the fluid in the creft

the deformed states were compared with the reference state at $t=0$.

\subsection{Strain calculations}

Figure 6 shows the maximum shear strain for the different regions of the matrix defined by Fig. 2b. The global maximum shear strain has an average and standard deviation significantly larger than the regions Matrix 1, Matrix 2 and 


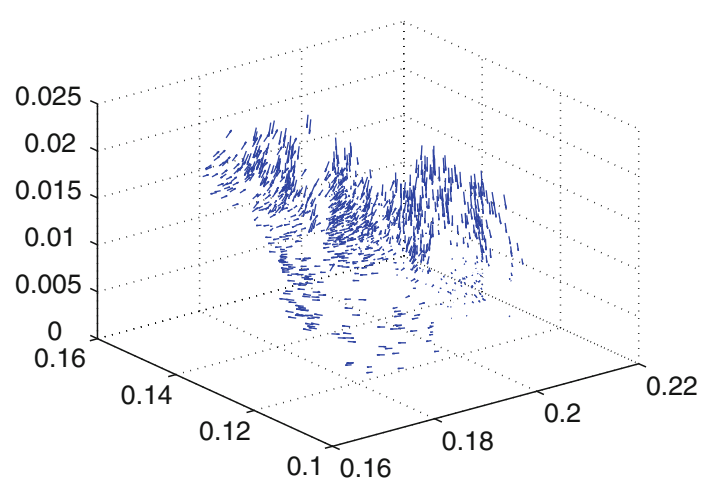

(a)

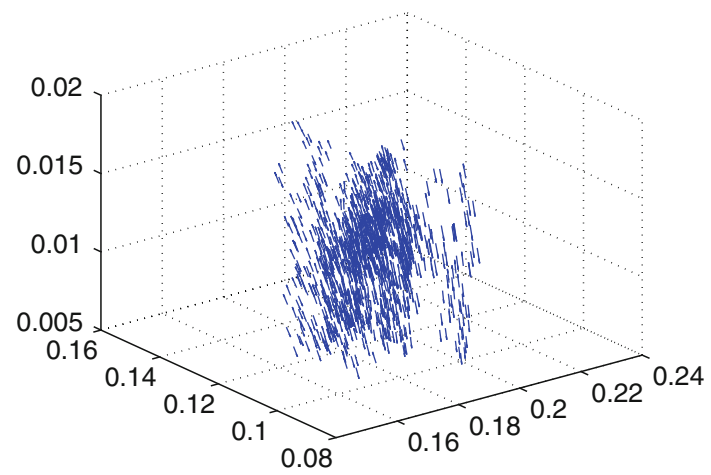

(b)

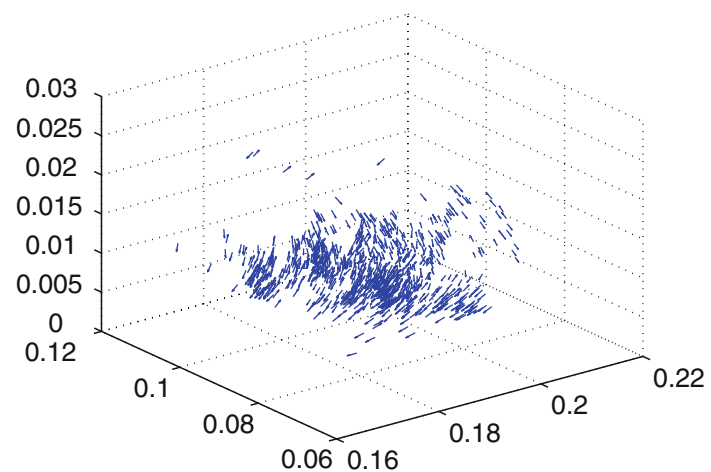

(c)

Fig. 8 First principal strain field in the extra-cellular matrix (region matrix 1) at time $t=60,120$ and $200 \mathrm{~min}$

Matrix 3. Similar results are obtained for the volume change of the tissue (Fig. 7) The results of the strain fields (Figs. 8, 9) of the local region matrix 1 over time show the heterogeneous character of the strain field changing in time. The first principal strain fields (Figs. 8, 9) show shift in size of the principal strain as well as in principal strain direction. To compute the total deformation in the sample, calculations were done on the global matrix grid as shown in Figs. $2 \mathrm{~b}$ and 5. The calculations of the strains at the global matrix level show an increase in the volume, while the volume change of Matrix 1, Matrix 2 and Matrix 3 are not significantly different from zero.

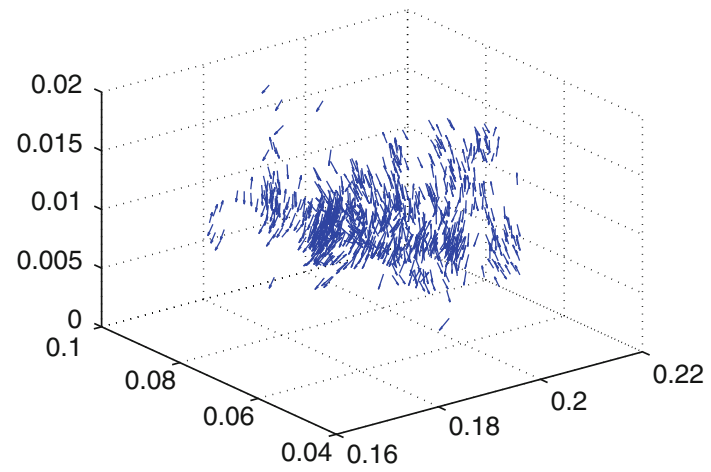

(a)

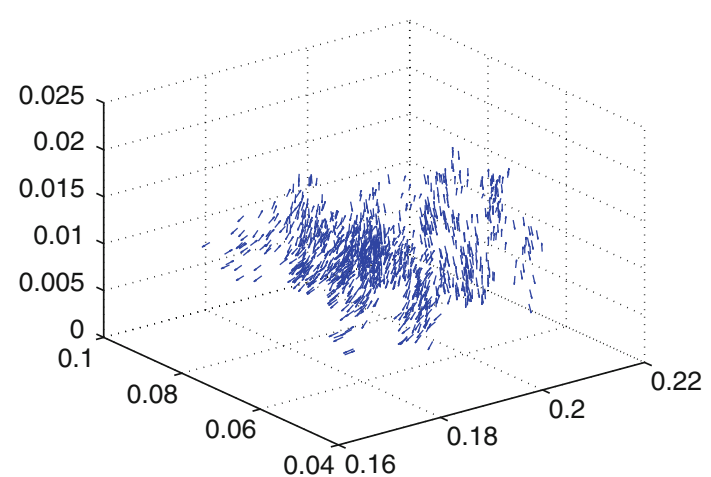

(b)

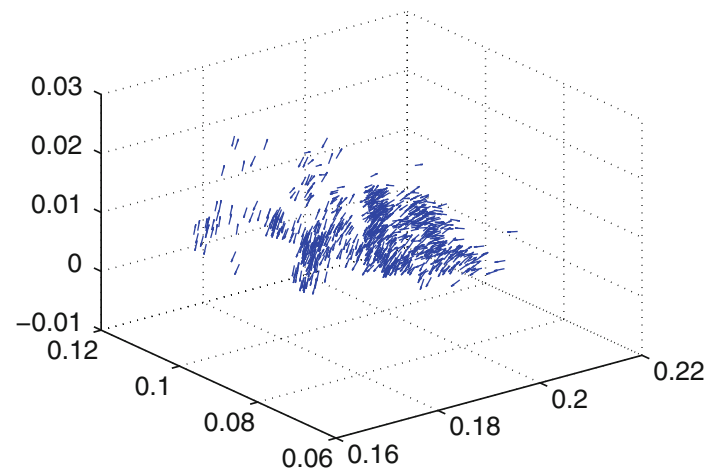

(c)

Fig. 9 First principal strain field in the extra-cellular matrix (region matrix 1) at time $t=300,400$ and $500 \mathrm{~min}$

\section{Discussion}

Three-dimensional finite strain of extra-cellular matrix samples of bovine annulus fibrosus tissue is measured under physiological osmotic prestressing. The microdeformation of the annulus tissue is strongly heterogeneous. The 3D correlation technique calculates $3 \mathrm{D}$ strain in collagen-rich areas. The results shown in this study are small in number. Hence, only trends and no definite conclusions can be drawn from this study. The results indicate that changes in external prestressing induce strongly non-affine deformation of the 
Fig. 10 Z-stack of an annulus fibrosus sample. a Z-stack images with $10 \%$ PEG, $0.15 \mathrm{M}$ $\mathrm{NaCl}$. b Z-stack images with $0 \%$ PEG, $0.15 \mathrm{M} \mathrm{NaCl}$
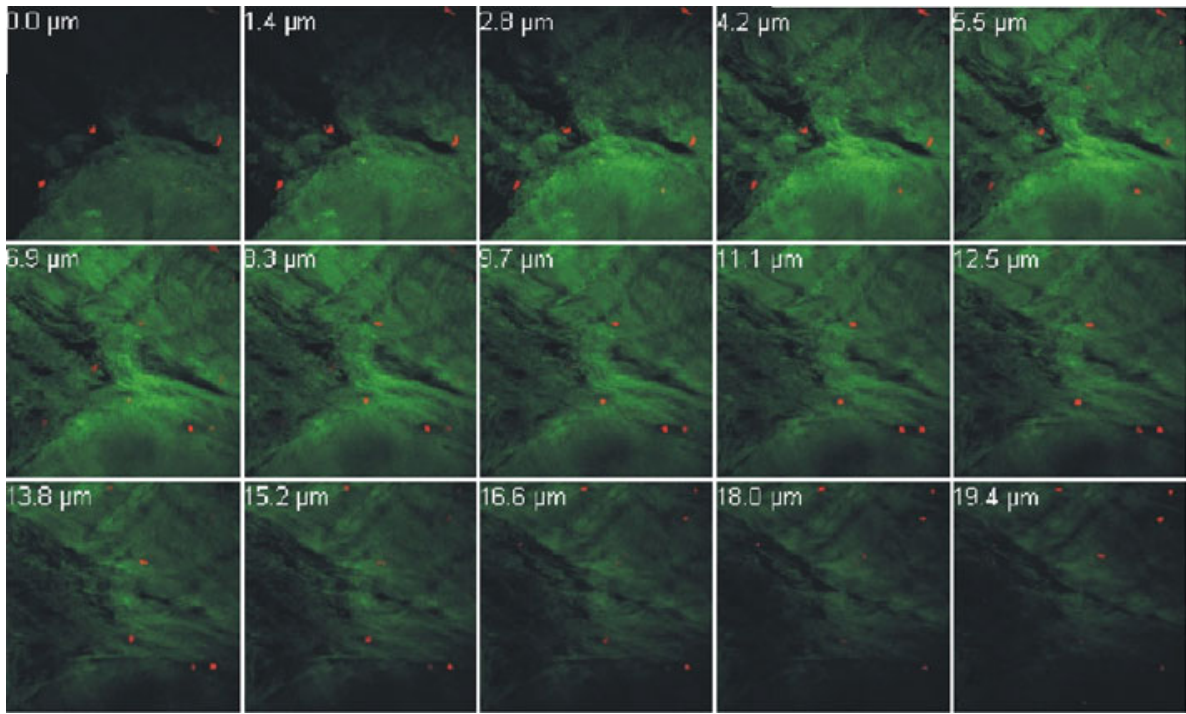

(a)
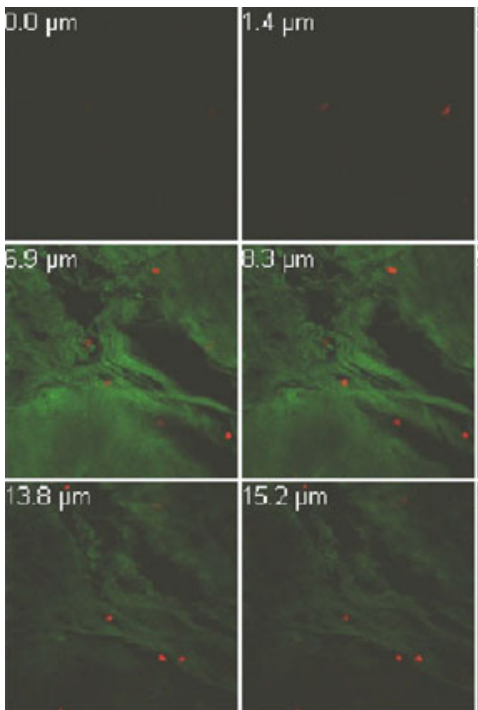
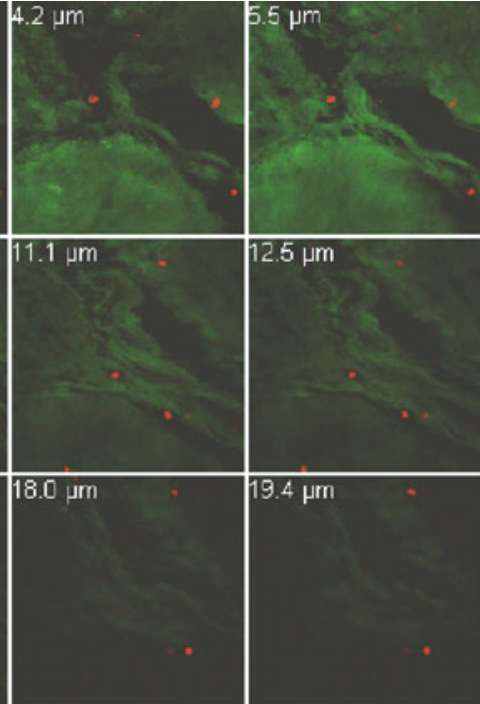

(b)

tissue. Macroscopic strains are generally not a good evaluation of microscopic strains around clefts and cells. This finding is consistent with findings from 2D-strains measured by Bruelmann et al. (2004b) and consistent with findings in the meniscus (Upton et al. 2008). Interlamellar strains seem larger than intralamellar strains. The microstrains cannot be inferred from a finite element analysis that deals with average strains and stresses only. Duncan and coworkers have demonstrated 2D shearing of collagen fibrils in the bovine disc (Bruelmann et al. 2004a,b). The present results indicate large normal strain between the fibrils on top of shearing (Figs. 10, 11).

The correlation technique used here was originally made for and tested on micro-computed tomography which has the same resolution in all directions. Because our images have been made with a confocal microscope, the resolution in the $z$-direction $(2.1 \mu \mathrm{m})$ is lower than in the $x$ - and $y$-direction $(0.4 \mu \mathrm{m})$. This affects the strain resolution in the $z$-direction. The experiments reconstructing a rigid body motion (Fig. 3) show that the image correlation is sufficient in the $x$ - and $y$-direction. Rotational and ridge displacements in the $z$-direction were not tested because of the limitation of the set-up. The correlation technique managed to calculate smooth $z$-displacements only after optimization of the subset size and search area. The lower $z$-resolution and the limited number of z-stacks (21 against 512 in the other directions) were probably the cause of the difficulty (Figs. 12, 13). 


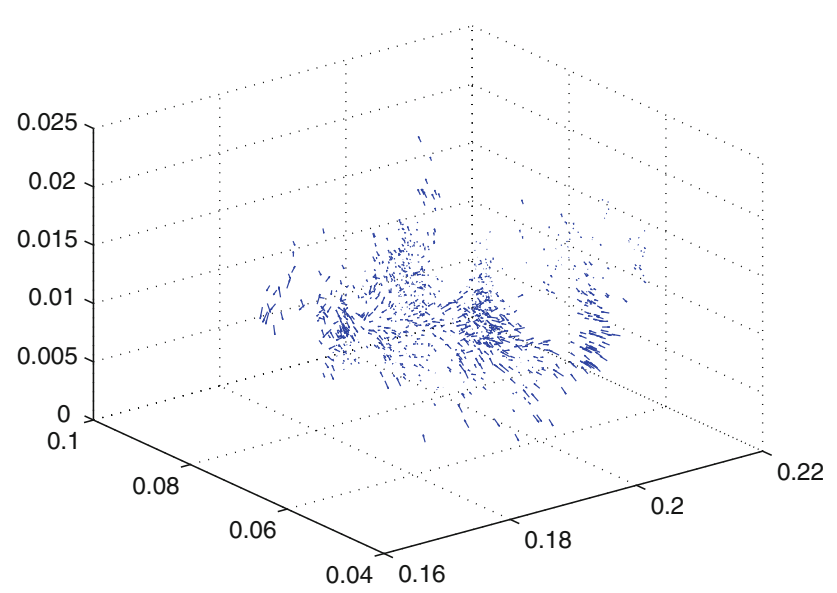

Fig. 11 2nd principal strain, matrix 1

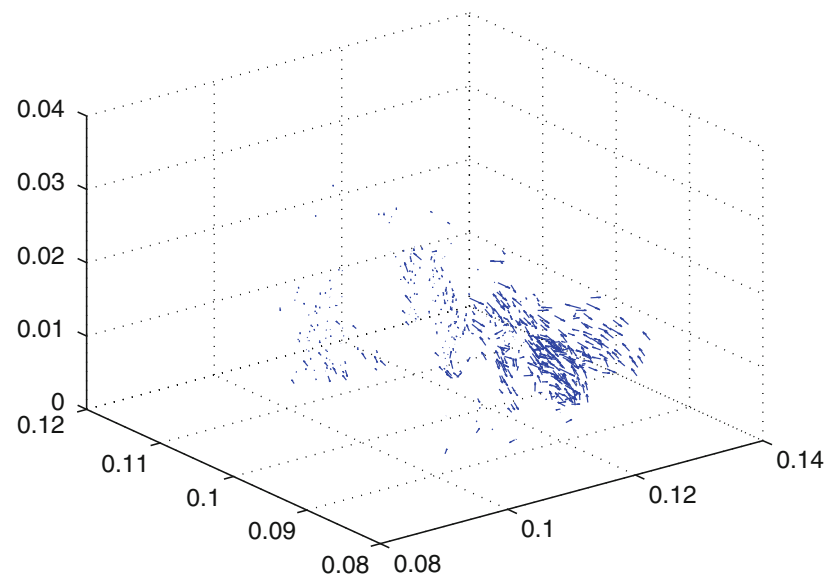

Fig. 12 2nd principal strain, matrix 2

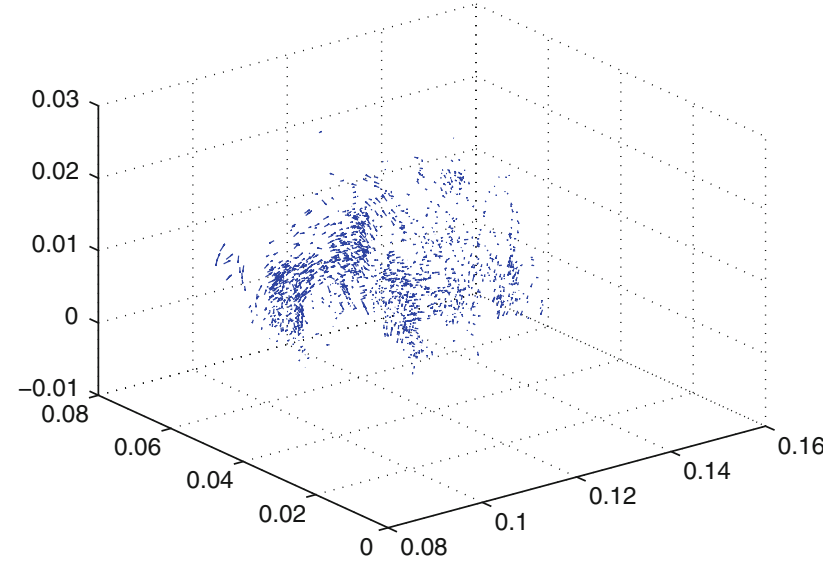

Fig. 13 2nd principal strain, matrix 3

In this study, samples stored at $-80^{\circ} \mathrm{C}$ were chosen. The ensuing necrosis of cells may induce an artefact to the deformation fields, particularly close to the dead cells. As the cell

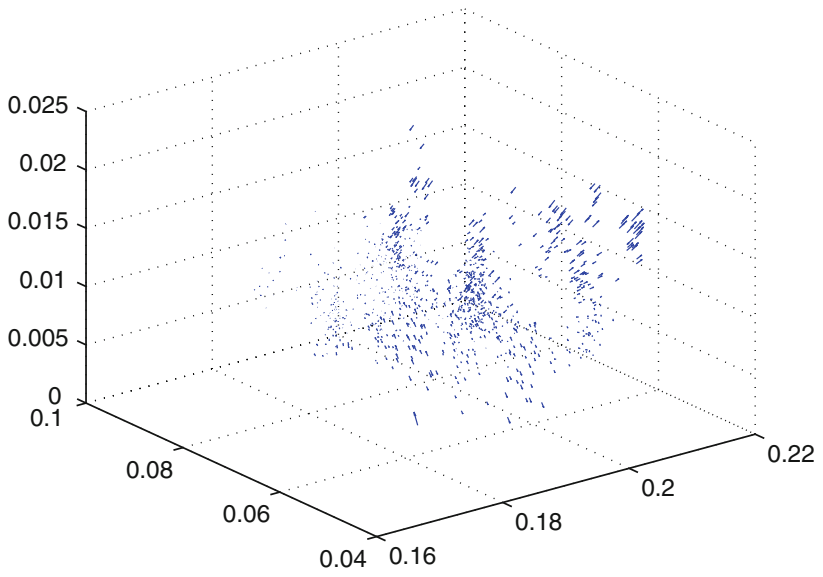

Fig. 14 3rd principal strain, matrix 1

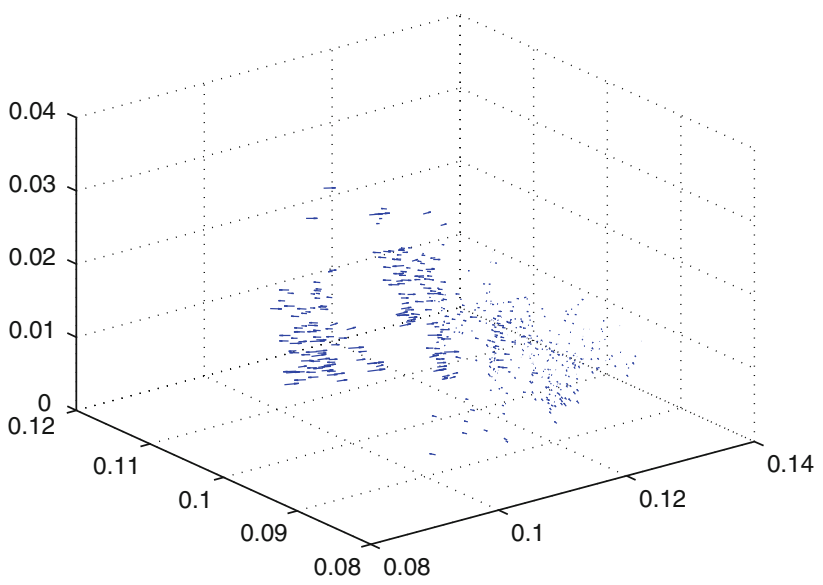

Fig. 15 3rd principal strain, matrix 2

stiffness is at least one order of magnitude lower than the stiffness of the peri- and extra-cellular matrix, the influence of the state of the cells on the strain patterns may be limited. From de Saint Venant's principle, the influence of the necrotic state of the cells should reaches up to a distance from the cells, equal to the diameter of the cells themselves. Future study will address this uncertainty by conducting similar experiments on living samples. The physiological osmotic prestressing as designed in this set-up should yield physiological hydration both for the cells as for the tissue (Figs. 14, 15).

A multiscale approach to intervertebral disc mechanics seems mandatory, if predictions of local strains around cells and discontinuities in the collagen structure are desired. While the sensitivity of intervertebral disc cells to mechanical stimuli is a well-established fact, the exact strains, stresses, pressure and osmotic pressures to which they are exposed are still open questions (Fig. 16). 


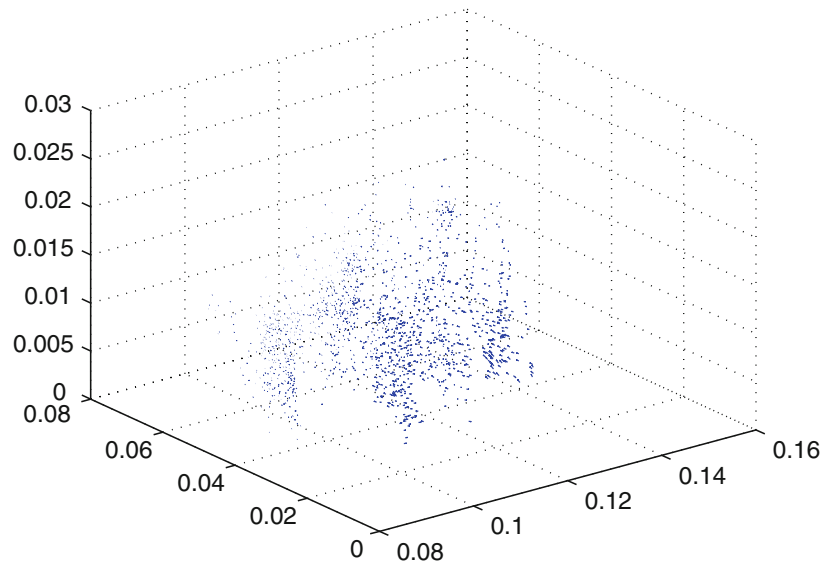

Fig. 16 3rd principal strain, matrix 3

Acknowledgments The research leading to these results has received funding from the European Community's Seventh Framework Programme (FP7, 2007-2013) under grant agreement no. HEALTH-F22008-201626.

Open Access This article is distributed under the terms of the Creative Commons Attribution Noncommercial License which permits any noncommercial use, distribution, and reproduction in any medium, provided the original author(s) and source are credited.

\section{References}

Bass EC, Ashford FA, Segal MR, Lotz JC (2004) Biaxial testing of human annulus fibrosus and its implications for a constitutive formulation. Ann Biomed Eng 32:1231-1242

Basser PJ, Schneiderman R, Bank RA, Wachtel E, Maroudas A (1998) Mechanical properties of the collagen network in human articular cartilage as measured by osmotic stress technique. Arch Biochem Biophys 351:207-219

Best BA, Guilak F, Setton LA, Zu W, Saed-Nejad F, Ratcliffe A, Weidenbaum M, Mow VC (1994) Compressive mechanical properties of the human anulus fibrosis and their relationship to biochemical composition. Spine 19(2):212-221

Bruelmann SB, Matyas JR, Duncan NA (2004) In situ intercellular mechanics of the bovine outer annulus fibrosus subjected to biaxial strains. J Biomech 37:223-231

Bruelmann SB, Matyas JR, Duncan NA (2004) Issls prize winner: collagen fibril sliding governs cell mechanics in the annulus fibrosus - an in situ confocal microscopy study of bovine discs. Spine 29:2612-2620

Hendriks F (2005) Mechanical behaviour of human epidermal and dermal layers in vivo. Ph.D. thesis, Eindhoven University of Technology, The Netherlands

Heuer F, Schmitt H, Schmidt H, Wilke HJ (2007) Creep associated changes in intervertebral disc bulging obtained with a laser scanning device. Clin Biomech 22:737-744

Houben GB, Drost MR, Huyghe JM, Janssen JD, Huson A (1997) Nonhomogeneous permeability of canine anulus fibrosus. Spine 22: $7-16$

Huyghe JM, Houben GB, Drost MR, van Donkelaar CC (2003) An ionised/non-ionised dual porosity model of intervertebral disc tissue: experimental quantification of parameters. Biomech Model mechanobiol 2:3-19
Iatridis JC, Laible JP, Krag MH (2003) Influence of fixed charge density magnitude and distribution on the intervertebral disc: applications of a poroelastic and chemical ellectric (peace) model. J Biomech Eng 125:12-24

Ishihara H, McNally DS, Urban J, Hall AC (1996) Effects of hydrostatic pressure on matrix synthesis in different regions of the intervertebral disk. J Appl Physiol 80:839-846

Juskaitis R (2006) Measuring the real point spread function of high numerical aperture microscope objective lenses. In: Pawley JB (ed) Handbook of biological confocal microscopy, 3rd edn, pp 239-250

Krahn KN, Bouten CVC, van Tuijl S, van Zandvoort MAMJ, Merkx M (2006) Fluorescently labeled collagen binding proteins allow specific visualisation of collagen in tissues and live cell culture. Anal Biochem 350:177-185

Maroudas A, Wachtel E, Grushko G, Katz EP, Weinberg P (1991) The effect of osmotic and mechanical pressures on water partitioning in articular cartilage. Biochim Biophys Acta 1073:285-294

Perie D, Korda D, Iatridis JC (2004) Confined compression experiments on bovine nucleus pulposus and annulus fibrosus: sensitivity of the experiment in the determination of compressive modulus and hydraulic permeability. J Biomech 38:2164-2171

Schroeder Y, Sivan S, Wilson W, Huyghe J, Maroudas A, Baaijens F (2007) Are disc pressure, stress and osmolarity affected by intra and extrafibrillar fluid exchange? J Orthop Res 25:1317-1324

Shirazi-Adl SA, Shrivastava SC, Ahmed AM (1984) Stress analysis of the lumbar disc-body unit in compression. Spine 9:120-134

Sivan S, Merkher Y, Wachtel E (2006) Correlation of swelling pressure and intrafibrillar water in young and aged human intervertebral discs. J Orthop Res 24:1292-1298

Sutton MA, Cheng M, Peters WH, McMeill SR (1986) Application of an optimized digital correlation method to planar deformation analysis. Image Vis Comput 4:143-150

Upton ML, Gilchrist CL, Guilak F, Setton LA (2008) Transfer of macroscale tissue strain to microscale cell regions in the deformed meniscus. Biophys J 95:2116-2124

Urban JPG, Maroudas A (1979) The measurement of fixed charge density in the intervertebral disc. Biochim Biophys Acta 586:166-178

Urban JPG, McMullin JF (1988) Swelling pressures of the lumbar intervertebral discs: influence of age, spinal level, composition and degeneration. Spine 13(2):179-187

Verhulp E, van Rietbergen B, Huiskes R (2004) A three-dimensional digital image coorelation technique for strain measurement in microstructures. J Biomech 37:1313-1320

Videman T, Nurmunen M (2004) The occurrence of annular tears and their relation to lifetime back pain history: a cadaveric study using barium sulfate discography. Spine 29:2668-2676

Wilke HJ, Neef P, Hinz B, Seidel R, Claes L (2001) Intradiscal pressure together with anthropometric data - a data set for the validation of models. Clin Biomech 16:S111-S126

Wognum S, Huyghe J, Baaijens F (2006) Influence of osmotic pressure changes on the opening of existing cracks in 2 intervertebral disc models. Spine 31:1783-1788

Wuertz C, Urban JP, Klasen J, Ignitius A, Wilke HJ, Claes LE, Neidlinger-Wilke C (2007) Influence of extracellular osmolarity and mechanical stimulation on gene expression of intervertebral disc cells. J Orthoped Res 11:1513-1522 